\title{
Numerical Study of Wave Propagation in Uniaxially Anisotropic Lorentzian Backward Wave Slabs
}

\author{
M.K. Kärkkäinen ${ }^{1}$ \\ ${ }^{1}$ Radio Laboratory, Helsinki University of Technology, P.O. Box 3000, FIN-02015 HUT, Finland
}

(Dated: October 3, 2018)

\begin{abstract}
The propagation and refraction of a cylindrical wave created by a line current through a slab of backward wave medium, also called left-handed medium, is numerically studied with FDTD. The slab is assumed to be uniaxially anisotropic. Several sets of constitutive parameters are considered and comparisons with theoretical results are made. Electric field distributions are studied inside and behind the slab. It is found that the shape of the wavefronts and the regions of real and complex wave vectors are in agreement with theoretical results.
\end{abstract}

PACS numbers: 02.70.Bf, 41.20.Jb, 77.22.Ch, 77.84.Lf

\section{INTRODUCTION}

Metamaterials have received much attention during the last years, because they possess unusual electromagnetic properties, like, for example, the opposite directions of phase and group velocities. Double negative (DNG) materials have negative permittivity and permeability, and they belong to the class of metamaterials. These media that are capable of supporting backward waves, have been also called backward wave (BW) media in the literature 1]. In BW media, the refraction phenomenon is anomalous in the sense that the power flow is refracted negatively, i.e. to the same side of the normal of the interface. As discussed in [1], it is not necessary for the medium to be a DNG medium to be able to support backward waves, because anomalous refraction can also be realized with anisotropic media with only one negative material parameter. We will use the term BW medium throughout the rest of the paper.

The pioneering work on BW materials by Veselago [2], where slab lenses were mentioned, has gained much attention during recent years, despite some differing opinions on the subject 3]. Isotropic BW materials are often called Veselago materials. The possibility to realize a perfect lens with isotropic BW slabs was discussed by Pendry in [4]. Numerical and theoretical considerations of wave propagation in isotropic BW slabs excited with a line current above the slab were presented by Ziolkowski and Heyman in 5. Guidance of waves in a slab of uniaxially anisotropic metamaterial has been theoretically discussed by Lindell and Ilvonen in [6] .

Controversial opinions regarding negative refraction effect and the perfect lens call for more detailed studies of the wave phenomena in backward-wave media. In this paper, wave propagation through uniaxially anisotropic BW slabs is numerically studied, and comparison is made with the theory presented in [1]. The theory predicts that there are regions in certain BW media, where the

*Electronic address: mkk@cc.hut.fi wave vector becomes complex, thus resulting in exponentially decaying waves. These regions are bounded by the asymptotes of the wave vector surfaces, which can be shown to be hyperbola. We study these phenomena numerically using the finite-difference time-domain (FDTD) method in a 2D-problem of a line current radiating in the vicinity of a BW slab. Also, the existence of surface waves on the interface between free space and BW medium is demonstrated with an example case. The BW medium is realized with Lorentzian constitutive parameters having a single pole pair.

An example problem with some theoretical discussion are presented in section 3. Results from the numerical simulations are shown and discussed in section 4. Our numerical simulations show that the wave propagation and refraction phenomena heavily depend on the parameter choices of the BW medium and are qualitatively in agreement with the theory.

\section{NUMERICAL MODEL OF DISPERSIVE MEDIUM}

The constitutive relations for a frequency dispersive isotropic medium read

$$
\mathbf{D}=\epsilon(\omega) \mathbf{E}, \quad \mathbf{B}=\mu(\omega) \mathbf{H}
$$

Negative permittivity and permeability are realized with the Lorentz medium model. The expressions for the permittivity and permeability are of the form

$$
\begin{aligned}
& \epsilon(\omega)=\epsilon_{0}\left(1+\frac{\omega_{p e}^{2}}{\omega_{0 e}^{2}-\omega^{2}+j \Gamma_{e} \omega}\right), \\
& \mu(\omega)=\mu_{0}\left(1+\frac{\omega_{p m}^{2}}{\omega_{0 m}^{2}-\omega^{2}+j \Gamma_{m} \omega}\right) .
\end{aligned}
$$

This model corresponds to a realization of BW materials as mixtures of conductive spirals or omega particles, as discussed in [7]. In this artificial material both electric and magnetic polarizations are due to currents induced 
on particles of only one shape, which provides a possibility to realize the same dispersion rule for both material parameters, as in (2). Note that the medium realized by Smith et. al. is built using different principles [8]. For the uniaxial materials that we consider in this paper we assume that the negative components of the material parameters are realized by small uniaxial spiral inclusions (racemic arrays with equal number of right- and left-handed particles) and possess frequency dispersion defined by (2). The positive components of the material parameters are equal to the free-space permittivity and permeability values, assuming that there are no particles oriented along these axes.

Equations (11) and (2) form the basis of the used FDTD model for BW materials. The two most important known FDTD methods for modeling dispersive materials like Lorentz materials are the recursive convolution method and the auxiliary differential equation method, where the constitutive parameters are expressed with the help of susceptibility. In the first method, $\mathbf{D}$ and $\mathbf{E}$ and $\mathbf{B}$ and $\mathbf{H}$ are related through a convolution integral. This approach is rather tedious. Another possibility is to use the auxiliary differential equation technique, which is slightly easier to implement. In this latter method, the polarization current associated to each Lorentz pole pair is introduced. These two models are discussed in detail in 9].

A third method to discretize fields in Lorentz medium, classified as direct integration method in [10], is based on the direct discretization of the PDE representing the time-domain equivalent of the simplified frequencydomain constitutive relation. The proposed discretization scheme is a modification of this method. The idea is to transform (11) into the time domain using the relation $j \omega \leftrightarrow \partial / \partial t$ with one integration before discretization using center differences. Usually, FDTD models based on the constitutive relation are directly (after multiplication with the denominator) discretized, as discussed in a summary of FDTD algorithms for dispersive media in [10]. We found in [11] that one integration prior to discretization leads to much better accuracy and, what is also important, to considerably better stability properties than the direct discretization without integration. In the numerical simulations, we have used the model which is discussed in detail in [1].

\section{AN EXAMPLE PROBLEM AND THEORETICAL DISCUSSION}

Consider a $z$-directed line current in free space located at a distance $d_{s}$ from a BW slab of thickness $d$. Let the interface between free space and the BW slab be located at $y=0$. The problem space is two-dimensional, with the field components $H_{x}, H_{y}$, and $E_{z}$. The peak of the incident spectrum is at $\omega_{p}=5.0 \cdot 10^{9} \mathrm{rad} / \mathrm{s}$, and the parameters in (2) are the following: $\omega_{0 e}=\omega_{0 m}=1.0 \cdot 10^{9}$ $\mathrm{rad} / \mathrm{s}, \omega_{p e}^{2}=\omega_{p m}^{2}=4.8 \cdot 10^{19}(\mathrm{rad} / \mathrm{s})^{2}, \Gamma_{e}=\Gamma_{m}=0$.
With these choices, we obtain $\epsilon(\omega)=\mu(\omega)$ for all $\omega$ and $\epsilon(\omega) / \epsilon_{0}=\mu(\omega) / \mu_{0}=-1$ at $\omega=\omega_{p}$. The spatial resolution $\Delta x=\Delta y=1.5 \mathrm{~cm}$ is used throughout the simulations. To be able to demonstrate the properties of BW materials, the incident spectrum is quite narrow, so that the relative constitutive parameters are close to minus one for the frequencies having significant spectral content. Absorbing boundary conditions are used to terminate the computational domain at the outer boundaries of the lattice. For simplicity, we have used Liao's third order ABC, although more sophisticated ABC's are available. The use of usual ABC's requires a small gap between the outer boundary of the computational space and the BW material slab. The chosen coordinate axes and the problem geometry is shown in Figure 1 Next, we

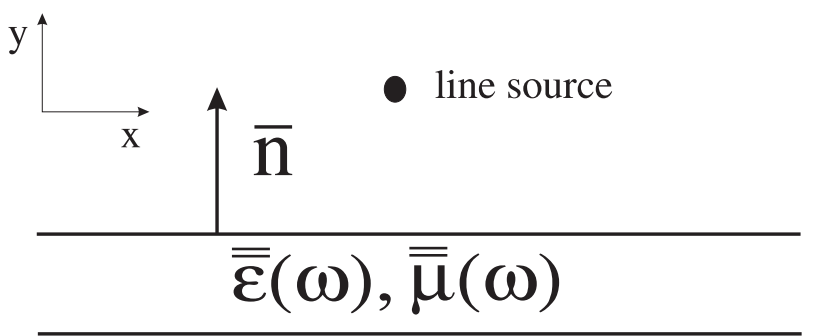

FIG. 1: The slab problem under consideration and the chosen coordinate system.

briefly present some theoretical results from [1] that are important here for comparison purposes with our numerical results. Wave propagation in BW slabs is studied with different value combinations of the medium parameters $\mu_{x}, \mu_{y}$ and $\epsilon_{z}$. For our TE-polarized case, the Poynting vector $\mathbf{S}_{T E}$ can be shown to read [1]

$$
\mathbf{S}_{T E}=\frac{\overline{\bar{\mu}} \cdot \mathbf{k}_{T E}}{2 k_{0} \eta_{0} \mu_{0} \mu_{x} \mu_{y}}\left|E_{0}\right|^{2},
$$

where $\left|E_{0}\right|$ is the amplitude of the TE-polarized electric field of the plane wave, $\mathbf{k}_{T E}$ is the wave vector with two cartesian components $k_{x}$ and $k_{y}, k_{0}=\omega \sqrt{\epsilon_{0} \mu_{0}}$ is the free space wave number, and $\eta_{0}=\sqrt{\mu_{0} / \epsilon_{0}}$ is the free space wave impedance. Denoting the angle between the outwards-pointing unit normal vector $\mathbf{u}_{y}$ and the wave vector $\mathbf{k}_{T E}$ by $\theta$, one can derive the dispersion equation and solve it for the wave number as in [1]. The result is

$$
k_{T E}(\theta)=k_{0} \sqrt{\frac{\mu_{x} \epsilon_{z}}{\cos ^{2} \theta+\frac{\mu_{x}}{\mu_{y}} \sin ^{2} \theta}} .
$$

For any given set of parameters, we may plot the projection curves of the wave vector surfaces in $x y$-plane. For certain choices of the medium parameters, the wave number becomes complex, resulting in exponentially decaying waves inside the BW material.

Any physical power flow must be directed downwards away from the source. This requires that

$$
\mathbf{u}_{y} \cdot \mathbf{S}_{T E}<0 .
$$


The necessary condition for any transmission is

$$
\frac{\mathbf{u}_{y} \cdot \mathbf{k}_{T E}}{\mu_{x}}<0 \Longleftrightarrow \frac{k_{y}}{\mu_{x}}<0 .
$$

Clearly, the phase velocity is directed oppositely to the power flow provided that $\mu_{x}<0$. Negative refraction of the Poynting vector requires that

$$
k_{x} \mathbf{u}_{x} \cdot \mathbf{S}_{T E}<0 \Longleftrightarrow \mu_{y}<0 .
$$

For the interface problem, we will also need to know conditions for the existence of surface waves. The input impedance on the surface filled by a uniaxial material is, for TE-polarized fields,

$$
Z_{\text {inp }}=\frac{\omega \mu_{x}}{\beta_{T E}},
$$

where the normal component of the propagation factor reads

$$
\beta_{T E}=\sqrt{\frac{\mu_{x}}{\mu_{y}}\left(\omega^{2} \epsilon_{z} \mu_{y}-k_{x}^{2}\right)}
$$

with the square root branch defined by $\operatorname{Im}\left(\beta_{T E}\right)<0 . k_{x}$ is the propagation factor along the surface. Thus, the eigensolutions for an interface between this medium and free space satisfy the following equation:

$$
\frac{\omega \mu_{x}}{\beta_{T E}}+\frac{\omega \mu_{0}}{\beta_{0}}=0
$$

where

$$
\beta_{0}=\sqrt{\omega^{2} \epsilon_{0} \mu_{0}-k_{x}^{2}}
$$

with $\operatorname{Im}\left(\beta_{0}\right)<0$.

Let us consider now conditions for the existence of surface waves along this interface. In this case both betas must be imaginary, of course with negative imaginary parts: $\beta_{T E}=-j \alpha_{T E}$ and $\beta_{0}=-j \alpha_{0}$, where $\alpha_{T E}>0$ and $\alpha_{0}>0$. The eigenvalue equation becomes

$$
\frac{\omega \mu_{x}}{\alpha_{T E}}+\frac{\omega \mu_{0}}{\alpha_{0}}=0
$$

Obviously, if all the material parameters are positive, this equation has no solutions, but if $\mu_{x}<0$, surface wave solutions are possible. This is well known for interfaces with free-electron plasma.

Five different cases will be considered in the following. In the first case, we choose $\mu_{x}>0, \mu_{y}<0$, and $\epsilon_{z}<0$. The wave vector surface is a two-sheeted hyperboloid with axis parallel to the $x$-axis. The asymptotes of the hyperbola in $x y$-plane divide the plane into regions of complex and real wave vectors. Waves that are propagating parallel to $y$-axis are supposed to decay exponentially inside the slab.

In the second case, $\mu_{x}>0, \mu_{y}<0, \epsilon_{z}>0$. The wave vector surface is a two-sheeted hyperboloid with the axis parallel to the surface normal. In our 2D-case, real wave vectors exist inside the region bounded by the asymptotes of the hyperbola associated to the wave vector surfaces.

As a third case, we consider the situation complementary to the second case in the sense that the signs of all the parameters are changed. Notice that this does not affect the shape of the wave vector curves. We have $\mu_{x}<0, \mu_{y}>0$, and $\epsilon_{z}<0$. In fact, the Poynting vector is refracted positively in this case (see (77)), but this is an interesting case anyway because of the aforementioned contrast with respect to the second case.

The fourth case consists of the usual isotropic BW slab ( $\mu_{x}=\mu_{y}<0$, and $\epsilon_{z}<0$ ) where focusing and negative refraction phenomena are present. The wave vector curves are ellipses (in our case of two equal parameters they are circles), and real wave vectors exist everywhere inside the slab.

The fifth case is specially chosen to show the existence of surface waves in the case when $\mu_{x}<0, \mu_{y}<0$, and $\epsilon_{z}>0$. We can easily see from (4) that there are neither real wave vectors nor backward waves. However, we have found that surface waves on the interface are easily excited in this case. Let us now present the numerical results.

\section{NUMERICAL RESULTS AND COMPARISON WITH THE THEORY}

In the first four cases, we show the electric field distribution at three suitable chosen increasing time steps to illustrate the wave propagation and refraction phenomena. In the fifth case, we illuminate a rectangular cylinder to see the surface waves. Whenever a constitutive parameter is said to be positive, it is supposed to be a constant and equal to the free space permittivity or permeability. Negative material parameters obey the Lorentzian dispersion rule and equal $-\epsilon_{0}$ or $-\mu_{0}$ at the center frequency.

A. Case I: $\mu_{x}>0, \mu_{y}<0, \epsilon_{z}<0$

In this case, the theory shows that the wave vectors are complex inside the slab within a region bounded by the asymptotes of a hyperbola. Inspection of Figure $2 \mathrm{c}$ ) reveals that there is indeed a region in the slab, where the electric field in negligible all the time. There are some fields within the slab just under the source. The hyperbola-shaped wavefronts propagate obliquely downwards inside the slab and the power flow is refracted negatively. The distance of the source from the first interface is discretized with 10 cells, and the thickness of the slab corresponds to 80 cells. 


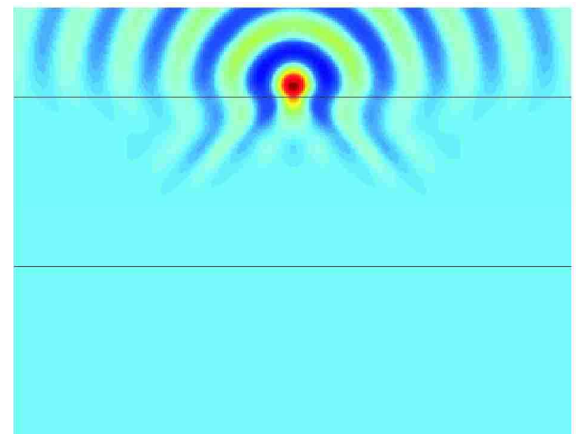

(a)

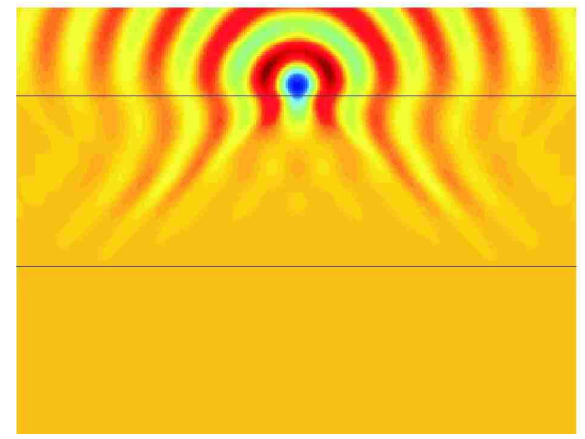

(b)

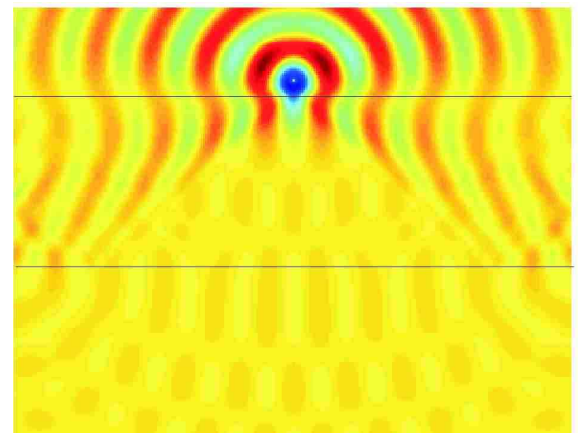

(c)

FIG. 2: (a) The electric field $E_{z}$ penetrating into the slab. Slab boundaries are indicated by black lines. (b) The electric field within the slab. Notice the region in the center of the slab, where the field amplitudes are very small. (c) It appears that the wavefronts inside the slab are hyperbolas, in agreement with the theory.

\section{B. Case II: $\mu_{x}>0, \mu_{y}<0, \epsilon_{z}>0$}

In Figure 3 a), a cylindrical wave is penetrating into the BW slab. In Figure 3 b), some numerical dispersion is visible. There are significant fields inside the region where the theory yields real wave vectors, while the fields are rather small elsewhere. Despite some dispersive effects, the wavefronts of constant field value are reminiscent of hyperbolas. The phase velocity is directed downwards. Some weak focusing of the power flow is seen in Figure $3 \mathrm{~b}$ ). The phase velocity inside the slab is directed downwards, as can be seen from the theory.

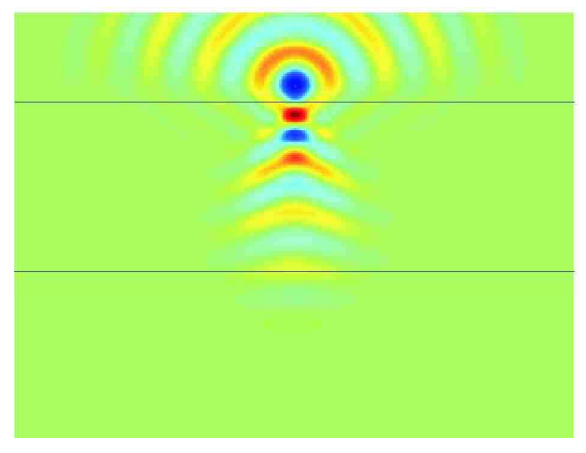

(a)

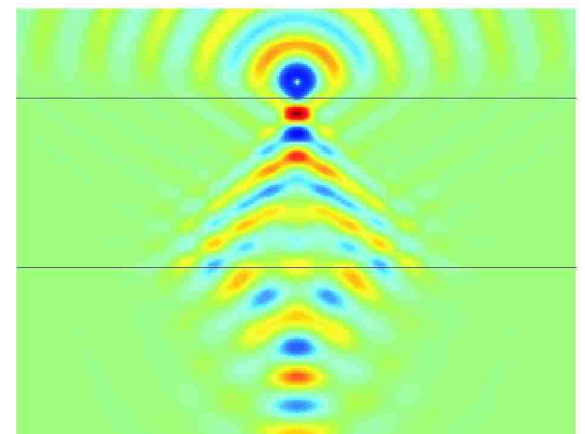

(b)

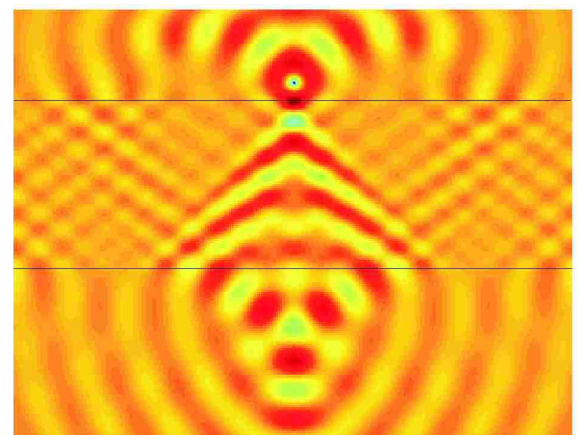

(c)

FIG. 3: (a) The electric field $E_{z}$ penetrating into the slab. (b) The electric field within the slab. Some waves have already passed through the slab. It is seen that there are small fields in the region, where the wave vector is complex, as predicted by the theory. (c) Dispersive effects are more clearly seen inside the slab. The electric field is concentrated to the lower side of the slab. The wavefronts behind the slab outside the region of large amplitudes are prolate ellipses. $d_{s}=10 \Delta y, d=80 \Delta y$.

C. Case III: $\mu_{x}<0, \mu_{y}>0, \epsilon_{z}<0$

To complete the analysis, we change the signs of the parameters of the second case. Clearly, the wave vector surfaces as defined by (4) are not changed. In fact, the Poynting vector is refracted positively in this case. However, the phase velocity is directed upwards [see (6) and (7)]. This phenomenon is clearly seen during the simulation. From Figure 4 we see that the electric field distributions inside the slab are quite similar to those of 
Figure 3 except that the wavefronts are less distorted in Figure 4 In Figure $4 \mathrm{c}$ ), the wavefronts behind the slab are seen to be oblate ellipses with the center on the lower interface of the slab.

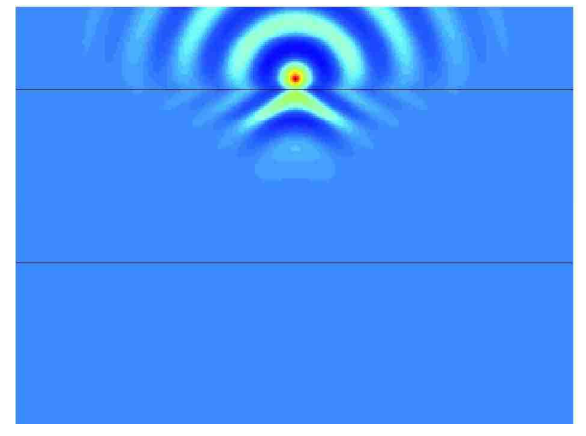

(a)

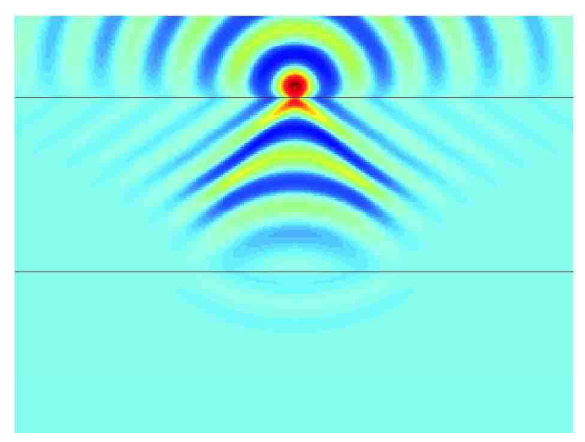

(b)

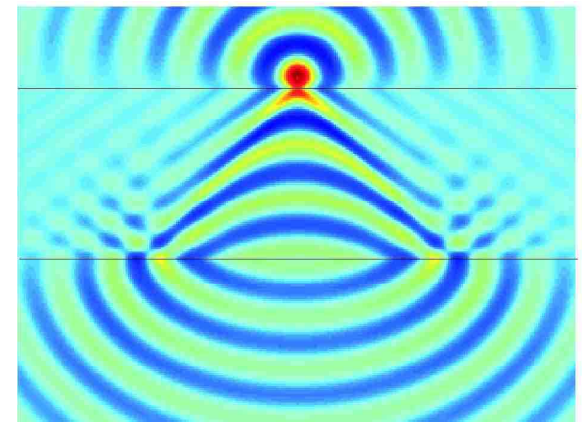

(c)

FIG. 4: (a) The electric field $E_{z}$ penetrating into the slab. Wavefronts are dramatically bent. (b) The electric field within the slab. Some waves have already passed through the slab. The wavefronts are seen to be hyperbolas. (c) Interestingly, the wavefronts behind the slab appear to be ellipses with the center on the lower interface of the slab. $d_{s}=10 \Delta y, d=80 \Delta y$

D. Case IV: an Isotropic Slab with $\mu_{x}<0, \mu_{y}<0$, $\epsilon_{z}<0$

Here we consider the usual isotropic BW (or double negative) slab with all the relative material parameters close to minus one. This case has been studied, for example, by Ziolkowski and Heyman in [5] in the case of Drude slabs. We obtained quite similar results with our alternative discretization technique in the case of isotropic Lorentz medium. The electric field distributions are shown in Figure [5] We can calculate the positions of the foci from the slab thickness $d$ and the distance $d_{s}$ of the source from the interface. Notice that we must have $d_{s}<d$ to have a focus inside the slab. The foci should appear at $y=-d_{s}$ inside the slab and at $y=-\left(2 d-d_{s}\right)$ behind the slab. The appropriate derivations can be found in 5$]$.

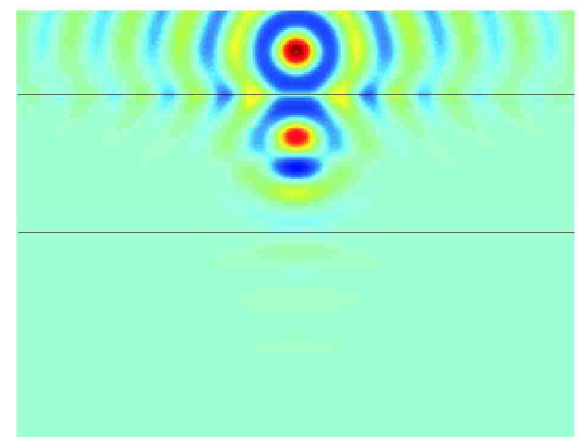

(a)

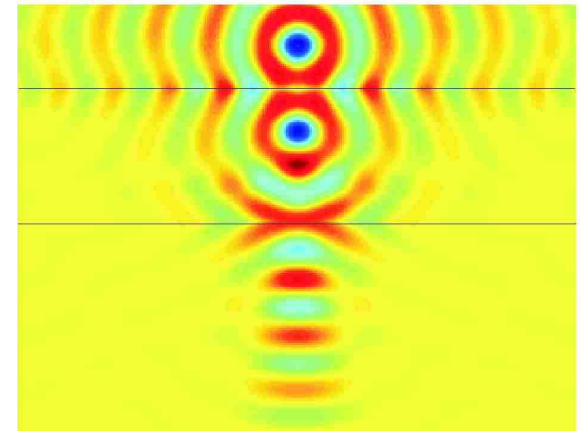

(b)

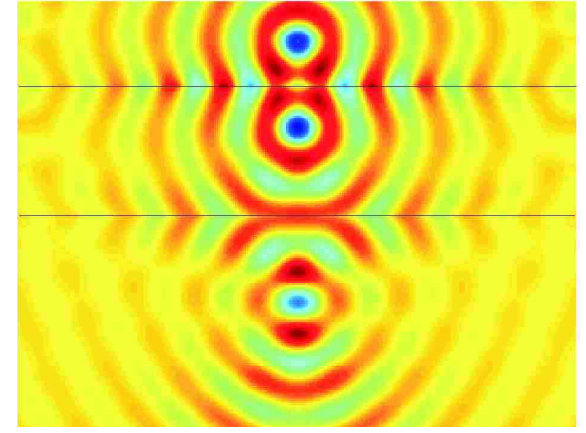

(c)

FIG. 5: (a) The electric field $E_{z}$ penetrating into an isotropic BW slab. (b) The electric field within the isotropic BW slab. Some waves have already passed through the slab. The first focus inside the slab is seen in the figure. (c) The second focus behind the BW slab has become visible. $d_{s}=20 \Delta y, d=60 \Delta y$ 
From Figure 5 a) we see that the electric field is concentrated in the expected position of the first focus. In Figure 5 c), the second focus behind the slab is also visible. It takes some time for the foci to develop, as is seen from Figure 5b), where the second focus is not yet seen. The incident spectrum has some small components for which the relative material parameters are not exactly minus unity. Hence the wavefronts are not perfect circles as predicted by the theory. Anyway, these results are in agreement with the theory concerning negative refraction of the Poynting vector. However, no "perfect" focusing has been observed, meaning that the focus area is always not smaller that about half wavelength. Steady state solutions for the foci were not obtained. This same observation was also made by Ziolkowski and Heyman in [5].

\section{E. Case V: $\mu_{x}<0, \mu_{y}<0, \epsilon_{z}>0$}

For this set of material parameters, the theory predicts that the waves decay exponentially everywhere inside the slab. However, we have found that surface waves are easily excited in this case, in accordance with the theoretical prediction, see siurface. To see this phenomenon, we illuminate a rectangular cylinder with a pulse having a slightly broader spectrum. The electric field distribution induced on the surface of the cylinder is shown in Figure 6 Figure [ a) shows how the surface waves begin

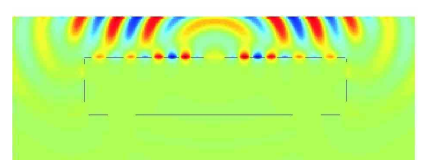

(a)

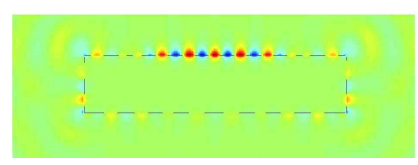

(b)
FIG. 6: (a) The surface waves begin to develop. The source is still on. (b) The source has been switched off, and surface waves exist around the cylinder. As is known from the theory, there are negligible fields inside the cylinder.

to develop. The source is still on. In Figure [ b b), the source above the cylinder has been switched off. The surface waves have propagated to the opposite side of the cylinder as well. In agreement with the theory, there are no fields inside the cylinder except for the immediate vicinity of the surface of the cylinder.

\section{CONCLUSIONS}

Wave propagation and refraction phenomena in uniaxially anisotropic BW slabs have been studied with the FDTD method. Special attention was paid to the shape of the wavefronts and to the regions inside the slabs, where the wave vector becomes complex, thus resulting in exponentially decaying waves. The numerical results for anisotropic BW slabs were seen to qualitatively agree well with the theoretical results. The effects of negative refraction, (imperfect) focusing, and surface wave excitation have been demonstrated. Potentially useful transformations of wave fronts between spherical, elliptical, and hyperbolical can be realized in homogeneous uniaxial backward wave slabs.

\section{Acknowledgments}

Financial support was received from Nokia Foundation and Emil Aaltonen Foundation. Useful discussions with S.A. Tretyakov, S.I. Maslovski and P.A. Belov are gratefully acknowledged.
[1] I. Lindell, S. Tretyakov, K. Nikoskinen, and S. Ilvonen, Microw. and Opt. Tech. Lett. 31, 129 (2001).

[2] V. Veselago, Sov. Phys. Uspekhi 10, 509 (1968).

[3] P. Valanju and R. Walser, Phys. Rev. Lett. 88, 187401 (2002).

[4] J. Pendry, Phys. Rev. Lett. 88, 3966 (2000).

[5] R. Ziolkowski and E. Heyman, Phys. Rev. E 53, 135 (2001).

[6] I. Lindell and S. Ilvonen, J. of Electromagn. Waves and Appl. 16, 303 (2002).

[7] S. Tretyakov, I. Nefedov, C. Simovski, and S. Maslovski, Advances in Electromagnetics of Complex Media and
Metamaterials (Kluwer, 2002).

[8] D. Smith, W. Padilla, D. Vier, S. Nemat-Nasser, and S. Schultz, Phys. Rev. Lett. 84, 4184 (2000).

[9] A. Taflove and S. Hagness, Computational Electrodynamics - The finite-difference time-domain method (Artech House, Boston, 2000).

[10] J. Young and R. Nelson, IEEE Antennas Propag. Magazine 43, 72 (2001).

[11] M. Kärkkäinen and S. Maslovski, Microw. and Opt. Tech. Lett. 5 (2003). 